\title{
Analytical comparison of the first- and second-order resonances for implementation of the dynamical Casimir effect in nonstationary circuit QED
}

\author{
E L S Silva ${ }^{1}$ and A V Dodonov ${ }^{1,2}$ \\ 1 Institute of Physics, University of Brasilia, 70910-900, Brasilia, Federal District, Brazil and \\ 2 International Center for Condensed Matter Physics, \\ University of Brasilia, 70910-900, Brasilia, Federal District, Brazil
}

\begin{abstract}
We investigate analytically and numerically the nonstationary circuit QED setup in which $N$ independent qubits interact with a single mode of the Electromagnetic field confined in a resonator. We consider the harmonic time modulation of some parameter (atomic transition frequency or the atom-field coupling strength) and derive the unitary dynamics up to the second order in the modulation depth for $N=1$ and $N \gg 1$. It is shown that all the resonant phenomena that occur for modulation frequencies $\sim 2 \omega_{0}$ (where $\omega_{0}$ is the cavity frequency) also occur for the halved frequencies. However, in the latter case the associated transition rates are significantly smaller and the modulation of the coupling strength is less effective. The transition rates are evaluated explicitly and the prospects of employing the second-order resonances in the phenomena related to the dynamical Casimir effect are examined.
\end{abstract}

PACS numbers: 42.50.Pq, 42.50.Ct, 42.50.Hz, 32.80-t, 03.65.Yz

\section{INTRODUCTION}

One of the direct proofs of the zero-point fluctuations predicted by quantum physics is the dynamical Casimir effect (DCE) - a rather broad term that denotes the creation of quanta from the vacuum state of some field due to fast changes of the geometry or internal properties of macroscopic or mesoscopic objects (see [1-4 for reviews). Although originally ascribed to the generation of photons due to the accelerated motion of a single mirror [5 7 or some cavity wall (where the photons can be accumulated [8 11]), this phenomenon has been extended to a variety of other systems, for instance, the phonon analogues in a Bose-Einstein condensate [12, 13, quantum fluid of light [14] or atomic gas with time-dependent effective charge [15. Recently two different groups implemented DCE analogues in the circuit Quantum Electrodynamics (QED) architecture [16 20], where the periodic motion of the boundary was simulated by driving magnetic flux through the SQUIDS (superconducting quantum interference devices) located at the end 21 or within the coplanar waveguide 22 .

It has been predicted [23 28] that the circuit QED architecture also allows for the implementation of DCE analogue using a single artificial atom - a macroscopic superconducting circuit composed of several Josephson junctions that has discrete energy levels, exhibits coherent quantum oscillations between these levels and can strongly couple to the electromagnetic field [20, 29 32. The internal properties of the artificial atom can be controlled in situ by applying external electric and magnetic fields [17, 20, 33 36. Hence, the generation of photons from vacuum via the modulation of parameters of a single two-level atom (qubit), also initiated in the ground state, would prove that DCE is an intrinsic effect of the light-matter interaction, originating from the counter-rotating terms in the interaction Hamiltonian. Indeed, within a toy model for DCE inside a cavity [26, the modulation of the material properties of a mesoscopic dielectric slab can be modeled by time-dependent transition frequencies of $N$ atoms, while the slab's oscillation can be viewed as varying atom-field coupling strengths.

The idea of using qubits with rapidly varying parameters goes beyond the simple verification of photon generation from vacuum due to the counter-rotating terms. By carefully adjusting the form of modulation, the intrinsic nonlinearities in the light-matter interaction [26, 37 may give rise to novel applications in quantum-information processing, quantum simulations and engineering of nonclassical states of light and matter 38 42]. Along with creation of photon pairs, one can engineer effective interactions that lead to simultaneous generation of photons and atomic excitations 23] or coherent annihilation of the system quanta from a known initial state [26, 27. An intuitive explanation for such behaviors is the modulation-induced selective coupling between the bare atom-field dressed states 25. The photon generation and annihilation rates can be enhanced by using a set of $N$ identical noninteracting qubits, when novel interaction regimes can be induced as a consequence of a richer spectrum of the composite system [26, 43, 45.

Although the qubit parameters can be readily tuned in the circuit QED setup [17, 20, 33, 36], one is still left with the technical difficulty of imposing a periodic modulation with frequency of the order $\eta \sim 2 \omega_{0}$, where $\omega_{0} / 2 \pi \sim 10 \mathrm{GHz}$ is the cavity frequency. So the goal of this paper is to discover whether one could use the halved modulation frequencies $\eta \sim \omega_{0}$ to achieve similar phenomena via the second-order resonances. It will be shown analytically in section [II that the answer is yes, and the modulation of the atomic transition frequency is more efficient than the modulation of the coupling strength to implement the second-order resonances, whereas for the first-order resonances they are of the 
same order [26, 27]. Moreover, in section III we shall perform numerical simulations in the presence of dissipation to assess whether the second-order resonances are feasible with the current parameters [37, 46, 47]. As demonstrated in A. analogous conclusions can be drawn for the case of $N \gg 1$ identical noninteracting qubits with time-dependent parameters. To find the main conclusions of the work the reader can skip directly to section IV.

\section{ANALYTICAL DESCRIPTION OF THE UNITARY DYNAMICS}

We consider a single mode of the electromagnetic field confined in a microwave waveguide resonator that interacts with $N$ identical qubits via the dipole interaction. Denoting the cavity frequency, the atomic transition frequency and the atom-field coupling parameter by $\omega_{0}, \Omega$ and $g$, respectively, the dynamics is governed by the generalized Rabi Hamiltonian [48, 50] (we set $\hbar=1$ )

$$
\hat{H}_{0}=\omega_{0} \hat{n}+i \chi_{0}\left(\hat{a}^{\dagger 2}-\hat{a}^{2}\right)+\sum_{l=1}^{N}\left[(\Omega / 2) \hat{\sigma}_{l, z}+g\left(\hat{a}+\hat{a}^{\dagger}\right)\left(\hat{\sigma}_{l,+}+\hat{\sigma}_{l,-}\right)\right] .
$$

Here $\hat{a}$ and $\hat{a}^{\dagger}$ are the bosonic ladder operators of the field and $\hat{n}=\hat{a}^{\dagger} \hat{a}$ is the photon number operator. The qubit operators are $\hat{\sigma}_{l,-}=\left|g_{l}\right\rangle\left\langle e_{l}\left|, \hat{\sigma}_{l,+}=\right| e_{l}\right\rangle\left\langle g_{l}\right|$ and $\hat{\sigma}_{l, z}=\left|e_{l}\right\rangle\left\langle e_{l}|-| g_{l}\right\rangle\left\langle g_{l}\right|$, where $\left|g_{l}\right\rangle$ and $\left|e_{l}\right\rangle$ denote the ground and excited states of the $l$-th qubit, respectively. The constant squeezing coefficient $\chi_{0}$ is due to the terms proportional to the square of the vector potential; it appears naturally when one uses the minimal-coupling Hamiltonian and the dipole approximation of the first order or higher [26, 51, 52. We keep this term for the sake of completeness, although it does not produce any new effect and its contribution is usually small. We assume that either $\Omega$ or $g$ is modulated externally as $X=X_{0}+\varepsilon_{X} \sin (\eta t)$, where $X=\{\Omega, g\}, \varepsilon_{X}$ is the corresponding modulation depth and $\eta$ is the angular frequency of modulation. The general description of the system dynamics under the first-order resonances was obtained in [25, 26] (valid even for the simultaneous multi-tone modulation of all the system parameters), so here we concentrate on the second-order effects whose mathematical analysis is more tricky.

For $N=1$ we work in the Schrödinger picture and expand the wavefunction corresponding to $\hat{H}_{0}$ as $[25]$

$$
|\psi(t)\rangle=e^{-i t \lambda_{0}} A_{0}(t)\left|\varphi_{0}\right\rangle+\sum_{n=1}^{\infty} \sum_{\mathcal{S}= \pm} e^{-i t \lambda_{n}, \mathcal{S}} A_{n, \mathcal{S}}(t)\left|\varphi_{n, \mathcal{S}}\right\rangle
$$

where $\lambda_{n, \mathcal{S}}$ and $\left|\varphi_{n, \mathcal{S}}\right\rangle$ are the $n$-excitations eigenvalues and eigenstates of the bare Jaynes-Cummings (JC) Hamiltonian 51 .

$$
\hat{H}_{J C}=\omega_{0} \hat{n}+\Omega_{0}|e\rangle\langle e|+g_{0}\left(\hat{a} \hat{\sigma}_{+}+\hat{a}^{\dagger} \hat{\sigma}_{-}\right)
$$

The index $\mathcal{S}$ labels the different eigenstates with the same number of excitations $n$.

The well known eigenfrequencies are

$$
\lambda_{0}=0, \lambda_{n>0, \mathcal{S}}=\omega_{0} n+\frac{\mathcal{S} \beta_{n}-\Delta_{-}}{2}, \beta_{n}=\sqrt{\Delta_{-}^{2}+4 g_{0}^{2} n}, \mathcal{S}= \pm,
$$

where $\Delta_{-}=\omega_{0}-\Omega_{0}$ is the average detuning. The JC eigenstates, also known as dressed states, are

$$
\left|\varphi_{0}\right\rangle=|g, 0\rangle,\left|\varphi_{n>0, \mathcal{S}}\right\rangle=\mathrm{s}_{n, \mathcal{S}}|g, n\rangle+\mathrm{c}_{n, \mathcal{S}}|e, n-1\rangle,
$$

where we introduced the notation

$$
\begin{gathered}
\mathrm{s}_{n,+}=\sin \theta_{n}, \mathrm{~s}_{n,-}=\cos \theta_{n}, \mathrm{c}_{n,+}=\cos \theta_{n}, \mathrm{c}_{n,-}=-\sin \theta_{n} \\
\theta_{n>0}=\arctan \frac{\Delta_{-}+\beta_{n}}{2 g_{0} \sqrt{n}} .
\end{gathered}
$$

We introduce effective time-dependent probability amplitudes $b(t)$ via the ansatz

$$
\begin{gathered}
A_{0}(t)=\exp \left(-i t \nu_{0}\right) b_{0}(t) \\
A_{m>0, \mathcal{T}}(t)=\exp \left(i \Pi_{m, \mathcal{T}, \mathcal{T}}^{(k)} \frac{\cos \eta t-1}{\eta}\right)\left[e^{-i t \nu_{m}, \mathcal{T}} b_{m, \mathcal{T}}(t)-\zeta_{m, \mathcal{T}}^{(k)}(t) e^{-i t \nu_{m,-\mathcal{T}}} b_{m,-\mathcal{T}}(t)\right]
\end{gathered}
$$


where $k=\{\Omega, g\}$. We defined the small time-dependent coefficient

$$
\begin{aligned}
\zeta_{m, \mathcal{T}}^{(k)}(t)= & \frac{i \Pi_{m, \mathcal{T},-\mathcal{T}}^{(k)} \exp \left(i \frac{\Pi_{m, \mathcal{T}, \mathcal{T}}^{(k)}-\Pi_{m,-\mathcal{T},-\mathcal{T}}^{(k)}}{2}\right)}{\eta} \\
& \times\left[\frac{e^{i t\left(\mathcal{T} \beta_{m}+\eta\right)}-1}{\eta+\mathcal{T} \beta_{m}}+\frac{e^{i t\left(\mathcal{T} \beta_{m}-\eta\right)}-1}{\eta-\mathcal{T} \beta_{m}}\right. \\
& \left.+\frac{\Pi_{m, \mathcal{T}, \mathcal{T}}^{(k)}-\Pi_{m,-\mathcal{T},-\mathcal{T}}^{(k)}}{2 i \eta}\left(\frac{e^{i t\left(\mathcal{T} \beta_{m}+2 \eta\right)}-1}{2 \eta+\mathcal{T} \beta_{m}}+\frac{e^{i t\left(\mathcal{T} \beta_{m}-2 \eta\right)}-1}{2 \eta-\mathcal{T} \beta_{m}}\right)\right]
\end{aligned}
$$

and the time-independent matrix elements

$$
\begin{gathered}
\Pi_{m, \mathcal{T}, \mathcal{S}}^{(k=\Omega)} \equiv \varepsilon_{\Omega}\left\langle\varphi_{m, \mathcal{T}} \mid e\right\rangle\left\langle e \mid \varphi_{m, \mathcal{S}}\right\rangle \\
\Pi_{m, \mathcal{T}, \mathcal{S}}^{(k=g)} \equiv \varepsilon_{g}\left\langle\varphi_{m, \mathcal{T}}\left|\left(\hat{a} \hat{\sigma}_{+}+\hat{a}^{\dagger} \hat{\sigma}_{-}\right)\right| \varphi_{m, \mathcal{S}}\right\rangle .
\end{gathered}
$$

These quantities can be calculated in a straightforward manner using the dressed states.

In equations (8) and (9) we included small 'intrinsic frequency shifts' $\nu$ - they appear due to the elimination of the rapidly oscillating terms and read [26]

$$
\begin{gathered}
\nu_{0}=-\sum_{\mathcal{S}= \pm} \frac{\mathrm{c}_{2, \mathcal{S}}^{2} g_{0}^{2}+2 \mathrm{~s}_{2, \mathcal{S}}^{2} \chi_{0}^{2}}{\lambda_{2, \mathcal{S}}} \\
\nu_{m>0, \mathcal{T}}=-g_{0}^{2} \sum_{\mathcal{S}= \pm}\left[\frac{\left|\overline{\boldsymbol{\Lambda}}_{m+2, \mathcal{T}, \mathcal{S}}\right|^{2}}{\lambda_{m+2, \mathcal{S}}-\lambda_{m, \mathcal{T}}}-\frac{\left|\overline{\boldsymbol{\Lambda}}_{m, \mathcal{S}, \mathcal{T}}\right|^{2}}{\lambda_{m, \mathcal{T}}-\lambda_{m-2, \mathcal{S}}}\right],
\end{gathered}
$$

where we defined

$$
\begin{gathered}
\bar{\Lambda}_{m+2, \mathcal{T}, \mathcal{S}} \equiv \Lambda_{m+2, \mathcal{T}, \mathcal{S}}-i \frac{\chi_{0}}{g_{0}} L_{m+2, \mathcal{T}, \mathcal{S}} \\
\Lambda_{m+2, \mathcal{T}, \mathcal{S}} \equiv\left\langle\varphi_{m, \mathcal{T}}\left|\hat{a} \hat{\sigma}_{-}\right| \varphi_{m+2, \mathcal{S}}\right\rangle, \quad L_{m+2, \mathcal{T}, \mathcal{S}} \equiv\left\langle\varphi_{m, \mathcal{T}}\left|\hat{a}^{2}\right| \varphi_{m+2, \mathcal{S}}\right\rangle .
\end{gathered}
$$

After long analytical manipulations in which the off-resonant terms were consistently eliminated through the rotating-wave approximation (RWA) [25], we find that the effective probability amplitudes obey the differential equation (to compact the notation we denote $b_{0, \mathcal{T}} \equiv b_{0},\left|\varphi_{0, \mathcal{T}}\right\rangle \equiv\left|\varphi_{0}\right\rangle$ and $\lambda_{0, \mathcal{T}} \equiv \lambda_{0}$ )

$$
\begin{aligned}
\dot{b}_{m, \mathcal{T}}= & \sum_{\mathcal{S}}\left[\left(\Theta_{m+2, \mathcal{T}, \mathcal{S}}^{(k)} e^{i t \eta}+\Phi_{m+2, \mathcal{T}, \mathcal{S}}^{(k)} e^{i t 2 \eta}\right) e^{-i t\left(\bar{\lambda}_{m+2, \mathcal{S}}-\bar{\lambda}_{m, \mathcal{T}}\right)} b_{m+2, \mathcal{S}}\right. \\
& \left.-\left(\Theta_{m, \mathcal{S}, \mathcal{T}}^{(k)} e^{i t \eta}+\Phi_{m, \mathcal{S}, \mathcal{T}}^{(k)} e^{i t 2 \eta}\right)^{*} e^{i t\left(\bar{\lambda}_{m, \mathcal{T}}-\bar{\lambda}_{m-2, \mathcal{S}}\right)} b_{m-2, \mathcal{S}}\right] .
\end{aligned}
$$

Hence both the first- and second-order resonances induce the transitions between the dressed states differing by two excitations. The time-independent transition rates corresponding to the first- and second-order resonances read

$$
\begin{aligned}
& \Theta_{m, \mathcal{T}, \mathcal{S}}^{(k)}=\frac{g_{0}}{2}\left[\sum_{\mathcal{R}= \pm}\left(\frac{\overline{\boldsymbol{\Lambda}}_{m, \mathcal{T}, \mathcal{R}} \Pi_{m, \mathcal{R}, \mathcal{S}}^{(k)}}{\eta-\mathcal{S} \delta_{\mathcal{R},-\mathcal{S}} \beta_{m}}-\frac{\overline{\boldsymbol{\Lambda}}_{m, \mathcal{R}, \mathcal{S}} \Pi_{m-2, \mathcal{T}, \mathcal{R}}^{(k)}}{\eta+\mathcal{T} \delta_{\mathcal{R},-\mathcal{T} \beta_{m-2}}}\right)-\frac{\delta_{k, g} \varepsilon_{g}}{g_{0}} \Lambda_{m, \mathcal{T}, \mathcal{S}}\right]
\end{aligned}
$$





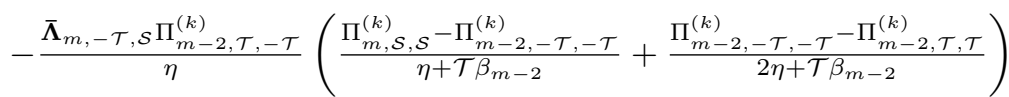

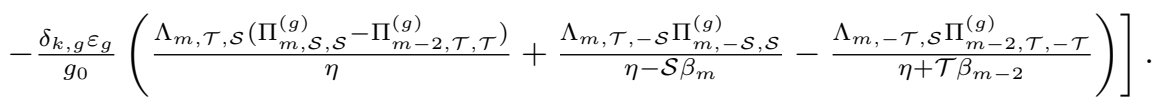


For the validity of equation (17) the following inequalities are required

$$
\begin{aligned}
& \left|\Pi_{m, \mathcal{S}, \mathcal{S}}^{(k)}-\Pi_{m,-\mathcal{S},-\mathcal{S}}^{(k)}\right|,\left|\Pi_{m, \mathcal{S},-\mathcal{S}}^{(k)}\right|,\left|\Pi_{m \pm 2, \mathcal{S}, \mathcal{S}}^{(k)}-\Pi_{m,-\mathcal{S},-\mathcal{S}}^{(k)}\right|, \\
& \left|\Pi_{m \pm 1, \mathcal{S}, \mathcal{S}}^{(k)}-\Pi_{m,-\mathcal{S},-\mathcal{S}}^{(k)}\right|,\left|g_{0} \Lambda_{m+2, \mathcal{T}, \mathcal{S}}\right|,\left|\chi_{0} L_{m+2, \mathcal{T}, \mathcal{S}}\right| \ll \omega_{0} .
\end{aligned}
$$

Notice that in equation (17) the resonant modulation frequencies correspond to the difference between two 'corrected' eigenfrequencies defined as

$$
\bar{\lambda}_{m, \mathcal{T}} \equiv \lambda_{m, \mathcal{T}}+\nu_{m, \mathcal{T}}+\Delta \nu_{m, \mathcal{T}}
$$

(we denote $\nu_{0, \mathcal{T}} \equiv \nu_{0}$ and $\bar{\lambda}_{0, \mathcal{T}} \equiv \bar{\lambda}_{0}$ ). So the Jaynes-Cummings eigenfrequencies are corrected by the terms $\nu_{m}, \mathcal{T}$ that include the standard Bloch-Siegert shift [53] and a small shift due to the squeezing coefficient. Within our approach we neglect the additional frequency shifts $\Delta \nu_{m, \mathcal{T}}$ of the order

$$
O\left(\Delta \nu_{m, \mathcal{T}}\right) \sim \frac{\left(\Pi_{m, \mathcal{S},-\mathcal{S}}^{(k)}\right)^{2}}{\omega_{0}}, \frac{\left(\Pi_{m \pm 2, \mathcal{S}, \mathcal{S}}^{(k)}-\Pi_{m,-\mathcal{S},-\mathcal{S}}^{(k)}\right)^{2}}{\omega_{0}}, k=\{\Omega, g\}
$$

and other shifts much smaller than $\nu_{m, \mathcal{T}}$. These terms were called 'systematic-error frequency shifts' (SEFS) in [26], since they appear due to the systematic simplification of the differential equations for $b_{m, \mathcal{T}}$ using the RWA [25]. The knowledge of SEFS is important because they slightly alter the resonant modulation frequencies, so ultimately they ought to be found numerically or experimentally to tune the exact resonance.

The functional dependence of the transition rates $\sqrt{18}$ and $\sqrt{19}$ on the system parameters is most clearly seen in the particular cases of the resonant and dispersive regimes studied below.

\section{A. Resonant regime}

For $\Delta_{-}=0$ the corrected eigenfrequencies are $\bar{\lambda}_{0}=-\left(\delta_{+}+\delta_{\chi} / 2\right)$ and $\bar{\lambda}_{m>0, \mathcal{S}}=\omega_{0} m+\mathcal{S} g_{0} \sqrt{m}-\left(\delta_{+}+m \delta_{\chi}\right)$, where $\delta_{ \pm} \equiv g_{0}^{2} / \Delta_{ \pm}, \delta_{\chi} \equiv 4 \chi_{0}^{2} / \Delta_{+}, \Delta_{+}=\omega_{0}+\Omega_{0}$. The criteria for validity of our method read: $\varepsilon_{\Omega}, g_{0} \sqrt{m}, \varepsilon_{g} \sqrt{m}$, $\chi_{0} m \ll \omega_{0}$ and $O\left(\Delta \nu_{m, \mathcal{T}}\right) \sim \varepsilon_{\Omega}^{2} / \omega_{0}, m \varepsilon_{g}^{2} / \omega_{0}$, where $m$ is the number of system excitations.

Using the JC eigenstates $\left|\varphi_{m>0, \mathcal{S}}\right\rangle=(|g, m\rangle+\mathcal{S}|e, m-1\rangle) / \sqrt{2}$ we obtain the following transition rates

$$
\begin{aligned}
\Theta_{2, \mathcal{T}, \mathcal{S}}^{(k=\Omega)} \simeq \mathcal{S} g_{0} \sqrt{2}\left(\frac{\varepsilon_{\Omega}}{8 \Omega_{0}}\right), \Phi_{2, \mathcal{T}, \mathcal{S}}^{(k=\Omega)} \simeq \mathcal{S} g_{0} \sqrt{2}\left(\frac{\varepsilon_{\Omega}}{8 \Omega_{0}}\right)^{2}\left(3 i-\mathcal{S} \sqrt{2} \frac{\chi_{0}}{g_{0}}\right) \\
\Theta_{m+2, \mathcal{T}, \mathcal{S}}^{(k=\Omega)} \simeq \mathcal{S} g_{0} \sqrt{m+1}\left(\frac{\varepsilon_{\Omega}}{8 \Omega_{0}}\right), \\
\Phi_{m+2, \mathcal{T}, \mathcal{S}}^{(k=\Omega)} \simeq \mathcal{S} g_{0} \sqrt{m+1}\left(\frac{\varepsilon_{\Omega}}{8 \Omega_{0}}\right)^{2}\left[2 i-\frac{2 \chi_{0}}{g_{0}}(\mathcal{S} \sqrt{m+2}+\mathcal{T} \sqrt{m})\right] \\
\Theta_{2, \mathcal{T}, \mathcal{S}}^{(k=g)} \simeq-\mathcal{S} \frac{g_{0}}{\sqrt{2}}\left(\frac{\varepsilon_{g}}{2 g_{0}}\right), \Phi_{2, \mathcal{T}, \mathcal{S}}^{(k=g)} \simeq-\mathcal{S} \frac{g_{0}}{\sqrt{2}}\left(\frac{\varepsilon_{g}}{2 g_{0}}\right)^{2} \frac{i \mathcal{S} \sqrt{2} g_{0}}{\omega_{0}} \\
\Theta_{m+2, \mathcal{T}, \mathcal{S}}^{(k=g)} \simeq-\mathcal{S} \frac{g_{0}}{2} \sqrt{m+1}\left(\frac{\varepsilon_{g}}{2 g_{0}}\right)^{2}, \\
\Phi_{m+2, \mathcal{T}, \mathcal{S}}^{(k=g)} \simeq-\mathcal{S} \frac{g_{0}}{2} \sqrt{m+1}\left(\frac{\varepsilon_{g}}{2 g_{0}}\right)^{2} \frac{i g_{0}}{\omega_{0}}(\mathcal{S} \sqrt{m+2}-\mathcal{T} \sqrt{m}),
\end{aligned}
$$

where $m>0$. Notice that for $k=\Omega$ the second-order resonances are one order of magnitude weaker than the first-order resonances, while for $k=g$ the transition rates are even smaller due to the additional factor $g_{0} / \omega_{0} \ll 1$. 


\section{B. Dispersive regime}

For $\left|\Delta_{-}\right| / 2 \gg g_{0} \sqrt{m}$ we obtain after expanding $\beta_{m}$ in equation (4)

$$
\begin{aligned}
\bar{\lambda}_{m, \mathcal{D}} & \simeq\left(\omega_{0}+\delta_{-}-\delta_{+}-\delta_{\chi}\right) m-\alpha m^{2}-\delta_{+}-\frac{1}{2} \delta_{\chi} \\
\bar{\lambda}_{m>0,-\mathcal{D}} & \simeq\left(\omega_{0}-\delta_{-}+\delta_{+}-\delta_{\chi}\right) m-\Delta_{-}+\alpha m^{2}-\delta_{+}+\frac{1}{2} \delta_{\chi},
\end{aligned}
$$

where we denote $\bar{\lambda}_{0, \mathcal{D}} \equiv \bar{\lambda}_{0} . \mathcal{D}=\Delta_{-} /\left|\Delta_{-}\right|= \pm$is the 'detuning symbol' and the effective single-photon Kerr nonlinearity strength is $\alpha=g_{0}^{4} / \Delta_{-}^{3}\left[26,53\right.$. To the first order in $g_{0} / \Delta_{-}$the JC eigenstates are

$$
\begin{aligned}
\left|\varphi_{m \geq 0, \mathcal{D}}\right\rangle & \simeq\left(|g, m\rangle+\frac{g_{0}}{\Delta_{-}} \sqrt{m}|e, m-1\rangle\right) \\
\left|\varphi_{m>0,-\mathcal{D}}\right\rangle & \simeq-\mathcal{D}\left(|e, m-1\rangle-\frac{g_{0}}{\Delta_{-}} \sqrt{m}|g, m\rangle\right)
\end{aligned}
$$

(we denote $\left|\varphi_{0, \mathcal{D}}\right\rangle \equiv\left|\varphi_{0}\right\rangle$ ) and the criteria for validity of our approach are the same as in section II A plus $\left|\Delta_{-}\right| \ll \omega_{0}$. In this regime one can distinguish three qualitatively different behaviors: the Anti-Jaynes-Cummings (AJC) behavior, DCE and Anti-DCE.

In the $A J C$ regime [23] one couples the states $\left\{\left|\varphi_{m, \mathcal{D}}\right\rangle,\left|\varphi_{m+2,-\mathcal{D}}\right\rangle\right\}$ when the modulation frequency is roughly $\eta \simeq \Delta_{+}$(1-order resonance) or $\eta \simeq \Delta_{+} / 2$ (2-order resonance). This corresponds roughly to the transition $|g, m\rangle \leftrightarrow$ $|e, m+1\rangle$, and the approximate expressions for the transition rates are (for $m \geq 0$ )

$$
\begin{gathered}
\Theta_{m+2, \mathcal{D},-\mathcal{D}}^{(k=\Omega)} \simeq-\mathcal{D} g_{0} \sqrt{m+1}\left(\frac{\varepsilon_{\Omega}}{2 \Delta_{+}}\right) \\
\Phi_{m+2, \mathcal{D},-\mathcal{D}}^{(k=\Omega)} \simeq-\mathcal{D} g_{0} \sqrt{m+1}\left(\frac{\varepsilon_{\Omega}}{2 \Delta_{+}}\right)^{2} \times 2 i \\
\Theta_{m+2, \mathcal{D},-\mathcal{D}}^{(k=g)} \simeq \mathcal{D} g_{0} \sqrt{m+1}\left(\frac{\varepsilon_{g}}{2 g_{0}}\right) \\
\Phi_{m+2, \mathcal{D},-\mathcal{D}}^{(k=g)} \simeq \mathcal{D} g_{0} \sqrt{m+1}\left(\frac{\varepsilon_{g}}{2 g_{0}}\right)^{2} \frac{g_{0}}{\Delta_{+}} \frac{4 g_{0}(m+1)}{i \Delta_{-}} .
\end{gathered}
$$

In the DCE behavior one couples the states $\left\{\left|\varphi_{m, \pm \mathcal{D}}\right\rangle,\left|\varphi_{m+2, \pm \mathcal{D}}\right\rangle,\left|\varphi_{m+4, \pm \mathcal{D}}\right\rangle, \ldots\right\}$ when the modulation frequency is close to $\eta \simeq 2 \omega_{0}$ or $\eta \simeq \omega_{0}$. Intuitively, these transitions correspond roughly to $|g, m\rangle \leftrightarrow|g, m+2\rangle \leftrightarrow|g, m+4\rangle \cdots$ for the states $(+\mathcal{D})$ or $|e, m\rangle \leftrightarrow|e, m+2\rangle \leftrightarrow|e, m+4\rangle \cdots$ for the states $(-\mathcal{D})$, so photon pairs can be generated from the initial vacuum field state (accompanied by low atomic excitation). A thorough analysis of the system dynamics under the 1-order resonance was performed in [26], where one showed that the average photon number and the degree of quadrature squeezing undergo saturation effects due to the effective Kerr nonlinearity, while the dynamics exhibits a collapse-revival behavior. The transition rates read $(m \geq 0)$

$$
\begin{aligned}
& \Theta_{m+2, \mathcal{D}, \mathcal{D}}^{(\Omega)} \simeq \delta_{-} \sqrt{(m+1)(m+2)}\left(\frac{\varepsilon_{\Omega}}{2 \Delta_{+}}\right) \\
& \Phi_{m+2, \mathcal{D}, \mathcal{D}}^{(\Omega)} \simeq \delta_{-} \sqrt{(m+1)(m+2)}\left(\frac{\varepsilon_{\Omega}}{2 \Delta_{+}}\right)^{2} \frac{\Delta_{+}}{\Omega_{0}}\left[i-\frac{2 \chi_{0}}{\Delta_{-}}(m+1)\right] \\
& \Theta_{m+2, \mathcal{D}, \mathcal{D}}^{(g)} \simeq-\delta_{-} \sqrt{(m+1)(m+2)}\left(\frac{\varepsilon_{g}}{2 g_{0}}\right) \frac{2 \Omega_{0}}{\Delta_{+}} \\
& \Phi_{m+2, \mathcal{D}, \mathcal{D}}^{(g)} \simeq-\delta_{-} \sqrt{(m+1)(m+2)}\left(\frac{\varepsilon_{g}}{2 g_{0}}\right)^{2} \frac{i \Delta_{-}}{\Omega_{0}} \\
& \Theta_{m+2,-\mathcal{D},-\mathcal{D}}^{(\Omega)} \simeq-\delta_{-} \sqrt{m(m+1)}\left(\frac{\varepsilon_{\Omega}}{2 \Delta_{+}}\right)^{2} \\
& \Phi_{m+2,-\mathcal{D},-\mathcal{D}}^{(\Omega)} \simeq-\delta_{-} \sqrt{m(m+1)}\left(\frac{\varepsilon_{\Omega}}{2 \Delta_{+}}\right)^{2} \frac{\Delta_{+}}{\Omega_{0}}\left[i+\frac{2 \chi_{0}}{\Delta_{-}}(m+1)\right]
\end{aligned}
$$




$$
\begin{aligned}
& \Theta_{m+2,-\mathcal{D},-\mathcal{D}}^{(g)} \simeq \delta_{-} \sqrt{m(m+1)}\left(\frac{\varepsilon_{g}}{2 g_{0}}\right) \frac{2 \Omega_{0}}{\Delta_{+}} \\
& \Phi_{m+2,-\mathcal{D},-\mathcal{D}}^{(g)} \simeq \delta_{-} \sqrt{m(m+1)}\left(\frac{\varepsilon_{g}}{2 g_{0}}\right)^{2} \frac{i \Delta_{-}}{\Omega_{0}} .
\end{aligned}
$$

The Anti-DCE behavior [26, 27, 45] couples the states $\left\{\left|\varphi_{m+2, \mathcal{D}}\right\rangle,\left|\varphi_{m,-\mathcal{D}}\right\rangle\right\}$ and occurs for $\eta \simeq\left(3 \omega_{0}-\Omega_{0}\right)$ or $\eta \simeq\left(3 \omega_{0}-\Omega_{0}\right) / 2$. Since this regime corresponds to the coherent annihilation of two excitations when the system is initiated in some state $(+\mathcal{D})$, roughly represented by the transition $|g, m+2\rangle \leftrightarrow|e, m-1\rangle$, the nickname Anti-DCE seems appropriate. The approximate transition rates are

$$
\begin{aligned}
\Theta_{m+2,-\mathcal{D}, \mathcal{D}}^{(\Omega)} & \simeq \frac{1}{2} \mathcal{D} \delta_{-} \frac{g_{0}}{\Delta_{-}} \sqrt{m(m+1)(m+2)}\left(\frac{\varepsilon_{\Omega}}{2 \omega_{0}}\right) \\
\Phi_{m+2,-\mathcal{D}, \mathcal{D}}^{(\Omega)} & \simeq \frac{1}{2} \mathcal{D} \delta_{-} \frac{g_{0}}{\Delta_{-}} \sqrt{m(m+1)(m+2)}\left(\frac{\varepsilon_{\Omega}}{2 \omega_{0}}\right)^{2}\left(i-\frac{2 \chi_{0}}{\Delta_{-}}\right) \\
\Theta_{m+2,-\mathcal{D}, \mathcal{D}}^{(g)} & \simeq-\mathcal{D} \delta_{-} \frac{g_{0}}{\Delta_{-}} \sqrt{m(m+1)(m+2)}\left(\frac{\varepsilon_{g}}{2 g_{0}}\right) \frac{\Omega_{0}}{\omega_{0}} \\
\Phi_{m+2,-\mathcal{D}, \mathcal{D}}^{(g)} & \simeq-\mathcal{D} \delta_{-} \frac{g_{0}}{\Delta_{-}} \sqrt{m(m+1)(m+2)}\left(\frac{\varepsilon_{g}}{2 g_{0}}\right)^{2} \frac{2 i \Delta_{-}}{\omega_{0}} .
\end{aligned}
$$

We notice that Anti-DCE is one order of magnitude weaker than DCE and only couples one pair of states, so it is harder to implement than all the other phenomena discussed above [27].

From equations $(31)$ - (38) we see that under the 2 -order resonances the modulation of $\Omega$ is more efficient than the modulation of $g$, although for the 1-order resonances both modulations give rise to transition rates with the same order of magnitude [assuming $O\left(\varepsilon_{\Omega} / \Omega_{0}\right) \sim O\left(\varepsilon_{g} / g_{0}\right)$ ].

\section{DISCUSSION}

In the preceding section we have evaluated explicitly the transition rates for single-qubit DCE and related phenomena under the 1- and 2-order resonances, showing that the modulation of $\Omega$ is more efficacious to achieve the second-order resonances. The first natural question that arises is whether such conclusion holds if one increases the number of qubits, e.g., by using a cloud of cold polar molecules trapped above the resonator [54, 55]. We treated this issue in the limit $N \gg 1$, when the Holstein-Primakoff transformation [56] permits to obtain relatively simple expressions for effective Hamiltonians. After long calculations summarized in A we confirmed that $\Omega$-modulation is again more efficient than $g$-modulation, and the associated transition rates have the functional dependence similar to the one found for $N=1$.

The second question is whether the second-order resonances are feasible with the current or near-future experimental parameters in the single-qubit circuit QED. Taking into account the realistic parameters $\omega_{0} / 2 \pi=10 \mathrm{GHz}, g_{0} / \omega_{0}=$ $5 \times 10^{-2}$ and $\Delta_{-}=0\left(\Delta_{-}=8 g_{0}\right)$ in the resonant (dispersive) regime [17, 19, 20, we shall calculate the transition rates for the $\Omega$-modulation assuming the perturbation depth $\varepsilon_{\Omega} / \Omega_{0}=5 \times 10^{-2}$ and setting $\chi_{0}=0$, recalling that for $g$-modulation the corresponding rates are much smaller. We shall denote the transition rates under the 1- and 2-order resonances by $\theta_{1} \equiv\left|\Theta_{M+2, \mathcal{T}, \mathcal{S}}^{(\Omega)}\right|$ and $\theta_{2} \equiv\left|\Phi_{M+2, \mathcal{T}, \mathcal{S}}^{(\Omega)}\right|$, where $M$ denotes the relevant number of the system excitations.

Since one of the main features of DCE is the photon generation from vacuum, we consider the initial zero-excitations state (ZES) $|g, 0\rangle$. In the resonant and AJC regimes we obtain $\theta_{1} / g_{0} \approx 9 \times 10^{-3}$ and $\theta_{2} / g_{0} \approx 2 \times 10^{-4}$, while for the DCE regime $\theta_{1} / g_{0} \approx 2 \times 10^{-3}$ and $\theta_{2} / g_{0} \approx 4 \times 10^{-5}$. Finally, for Anti-DCE and the initial state with $m \approx 5$ excitations we have $\theta_{1} / g_{0} \approx 3 \times 10^{-4}$ and $\theta_{2} / g_{0} \approx 4 \times 10^{-6}$. These values must be compared with the dominant dissipative parameters - the cavity (qubit) damping rate $\kappa(\gamma)$ and the qubit's pure dephasing rate $\gamma_{\phi}$. Considering the state-of-the-art parameters $\kappa \sim \gamma \sim \gamma_{\phi} \sim 5 \times 10^{-5} g_{0}$ [37, 46, 47, it seems that the photon generation from vacuum using the 2-order resonances is possible in systems with weak dissipation.

To estimate the actual behavior one has to solve the master equation for the density matrix

$$
d \hat{\rho} / d t=-i[\hat{H}, \hat{\rho}]+\hat{\mathcal{L}} \hat{\rho},
$$

where the Liouvillian superoperator $\hat{\mathcal{L}}$ depends on the system-reservoir interaction. We assume the Markovian regime and employ the standard master equation (SME) of Quantum Optics, since recent studies [27, 28] have shown that 
for the parameters considered here it reproduces quite well the results of a more sophisticated microscopic model [53]. At zero temperature our dissipative kernel reads [57]

$$
\hat{\mathcal{L}} \hat{\rho}=\kappa D[\hat{a}] \hat{\rho}+\gamma D\left[\hat{\sigma}_{-}\right] \hat{\rho}+\frac{\gamma_{\phi}}{2} D\left[\hat{\sigma}_{z}\right] \hat{\rho},
$$

where $D[\hat{O}] \hat{\rho} \equiv \frac{1}{2}\left(2 \hat{O} \hat{\rho} \hat{O}^{\dagger}-\hat{O}^{\dagger} \hat{O} \hat{\rho}-\hat{\rho} \hat{O}^{\dagger} \hat{O}\right)$ is the Lindbladian superoperator and $\hat{\sigma}_{z} \equiv|e\rangle\langle e|-| g\rangle\langle g|$. Typical dynamics under the 1- and 2-order resonances in the resonant, AJC and DCE regimes is displayed in figures 1 , 2 and 3. respectively, where equation (39) was solved numerically for the initial ZES. The plots illustrate the evolution of the average photon number $\langle\hat{n}\rangle$, the atomic excitation probability $P_{e}=\operatorname{Tr}[|e\rangle\langle e| \hat{\rho}]$ and the probability of the ZES $P_{\{g, 0\}}=\langle g, 0|\hat{\rho}| g, 0\rangle$ for parameters $g_{0} / \omega_{0}=5 \times 10^{-2}, \kappa=\gamma=\gamma_{\phi}=10^{-4} g_{0}, \Delta_{-}=0\left(\Delta_{-}=8 g_{0}\right)$ in the resonant (dispersive) regime, $\varepsilon_{\Omega} / \Omega_{0}=5 \times 10^{-2}$ in figures 1 -2 and $\varepsilon_{\Omega} / \Omega_{0}=10^{-1}$ in figure 3 . One can see that a measurable amount of cavity and atomic excitations can still be generated under the 2-order resonances on the timescale of a few microseconds, and the oscillatory behavior predicted by equation (17) is partially preserved. Moreover, the probability of measuring states other than the initial ZES can become larger than $70 \%$ for some time intervals.

$\mathrm{t}(\mu \mathrm{s})$

$\mathrm{t}(\mu \mathrm{s})$

$\mathrm{t}(\mu \mathrm{s})$
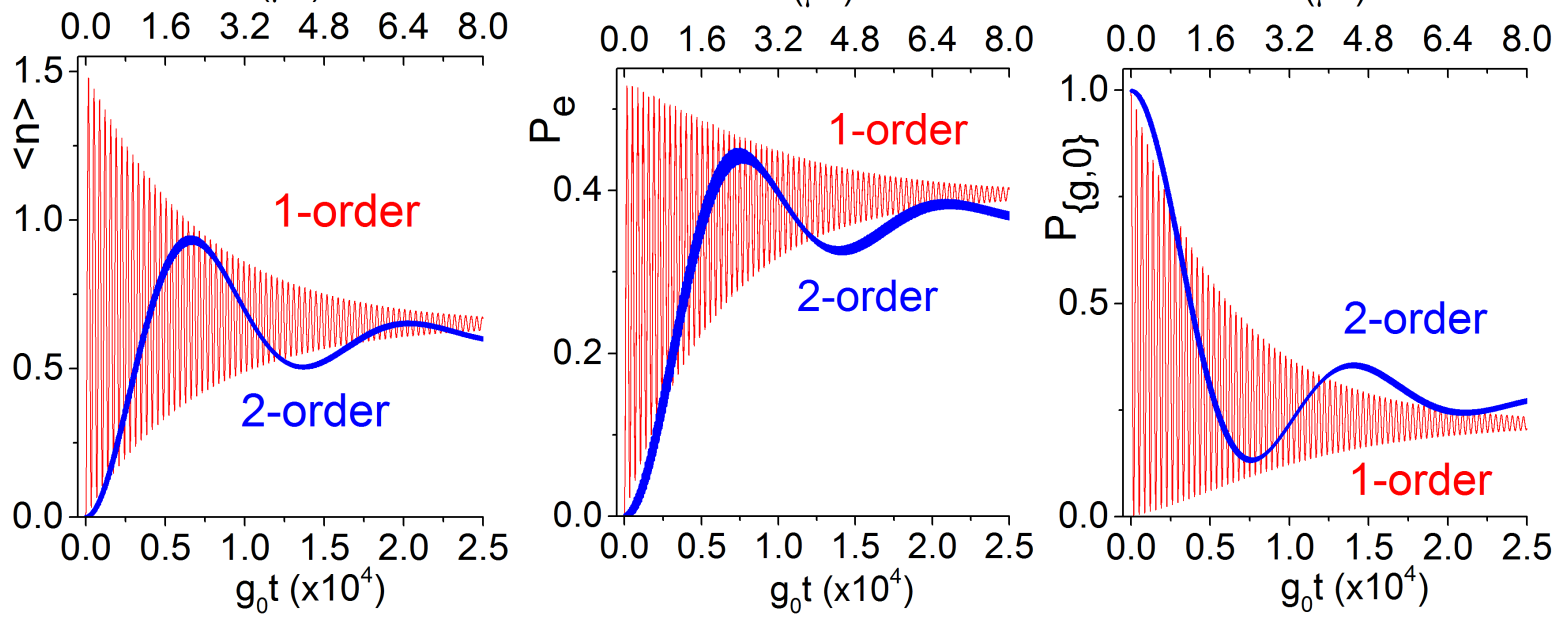

FIG. 1: (Color online) Dynamics of $\langle\hat{n}\rangle, P_{e}$ and $P_{\{g, 0\}}$ for photon generation from the ZES in the resonant regime. The resonant modulation frequencies found numerically are $\eta=\left(\lambda_{2,+}-\lambda_{0}-7 \times 10^{-2} \delta_{+}\right) / K$, where $K=1$ or $K=2$.

From figures 12 we see that, curiously, the asymptotic values are quite close under the 1- and 2-order resonances. To explain such behavior, one can derive closed analytical expressions by writing the differential equations for the density matrix elements in the dressed basis and performing RWA. Following the steps of [27, after long calculations we find that for the resonant regime (section IIA) and $\kappa=\gamma=\gamma_{\phi}$ we obtain for $t \rightarrow \infty$

$$
\langle\hat{n}\rangle^{(\infty)} \simeq \frac{15}{23+9(\gamma / \theta)^{2}}, P_{e}^{(\infty)} \simeq \frac{3}{5}\langle n\rangle_{\infty}, P_{\{g, 0\}}^{(\infty)} \simeq \frac{5+9(\gamma / \theta)^{2}}{23+9(\gamma / \theta)^{2}},
$$

where $\theta$ stands for $\theta_{1}$ or $\theta_{2}$, depending on the order of resonance. Another relevant scenario is the case of negligible cavity damping, $\kappa=0$, when for $\gamma=\gamma_{\phi}$

$$
\langle\hat{n}\rangle^{(\infty)} \simeq \frac{6}{8+(3 \gamma / 4 \theta)^{2}}, P_{e}^{(\infty)} \simeq \frac{1}{2}\langle n\rangle^{(\infty)}, P_{\{g, 0\}}^{(\infty)} \simeq \frac{2+(3 \gamma / 4 \theta)^{2}}{8+(3 \gamma / 4 \theta)^{2}} .
$$

Similarly, for the AJC regime (section IIB) we obtain for $\kappa=\gamma=\gamma_{\phi}$

$$
\langle\hat{n}\rangle^{(\infty)} \simeq P_{e}^{(\infty)} \simeq\left[2+(\gamma / \theta)^{2}\right]^{-1}, P_{\{g, 0\}}^{(\infty)} \simeq \frac{1}{2} \frac{1+2(\gamma / \theta)^{2}}{2+(\gamma / \theta)^{2}}
$$

and for $\kappa=0, \gamma=\gamma_{\phi}$

$$
\langle\hat{n}\rangle^{(\infty)} \simeq\left[1+\left(\frac{g_{0}}{\Delta_{-}}\right)^{2}\left(\frac{3 \gamma}{2 \theta}\right)^{2}\right]^{-1}, P_{e}^{(\infty)} \simeq 6\left(\frac{g_{0}}{\Delta_{-}}\right)^{2}\langle\hat{n}\rangle^{(\infty)}, \quad P_{\{g, 0\}}^{(\infty)} \simeq\left(\frac{g_{0}}{\Delta_{-}}\right)^{2} \frac{3+(3 \gamma / 2 \theta)^{2}}{1+\left(g_{0} / \Delta_{-}\right)^{2}(3 \gamma / 2 \theta)^{2}} .
$$


$\mathrm{t}(\mu \mathrm{s})$

$\mathrm{t}(\mu \mathrm{s})$

$\mathrm{t}(\mu \mathrm{s})$


FIG. 2: (Color online) Similar to figure1, but for the AJC regime. The resonant modulation frequencies are $\eta=\left(\lambda_{2,-\mathcal{D}}-\lambda_{0}+\right.$ $\left.1.916 \delta_{+}\right) / K$.

$\mathrm{t}(\mu \mathrm{s})$

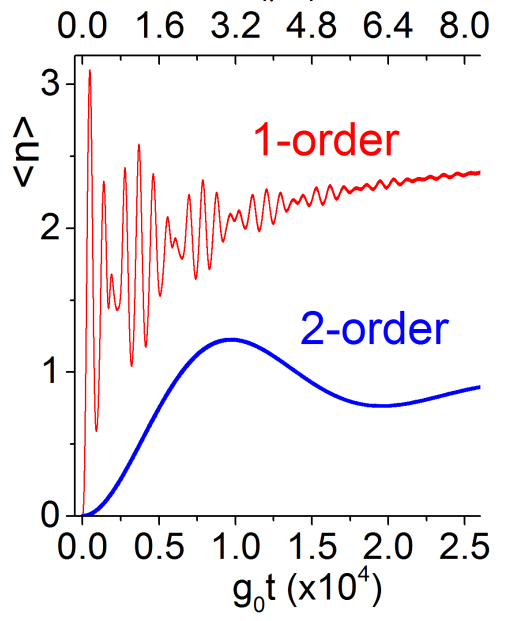

$\mathrm{t}(\mu \mathrm{s})$

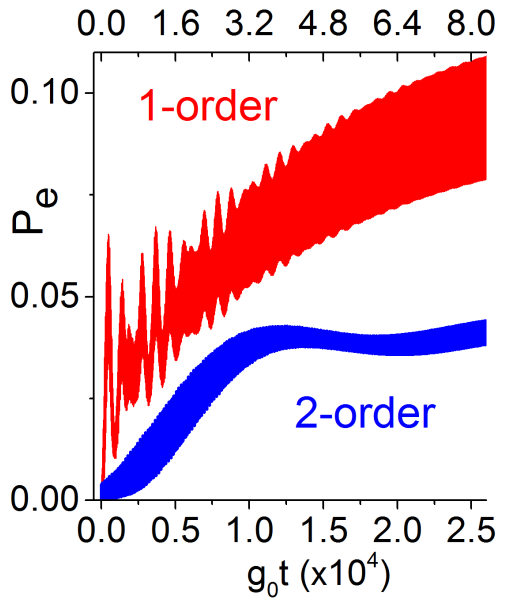

$\mathrm{t}(\mu \mathrm{s})$
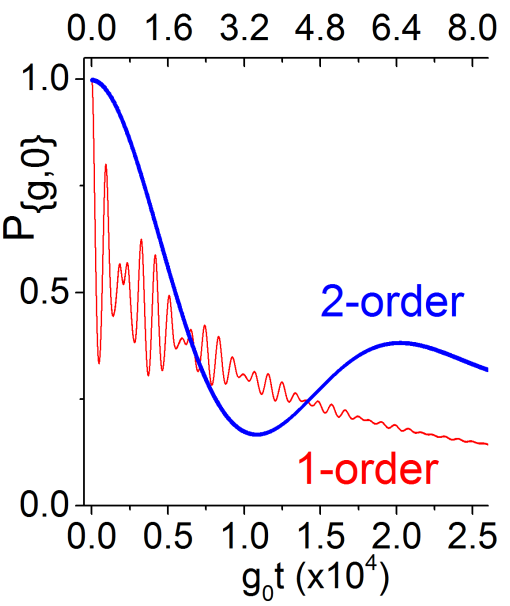

FIG. 3: (Color online) Similar to figure1 but for the DCE regime. The resonant modulation frequencies are $\eta=\left(\lambda_{2, \mathcal{D}}-\lambda_{0}-\right.$ $\left.1.93 \delta_{+}\right) / K, K=1,2$.

These expressions fit surprisingly well the numerical data and explain why the asymptotic values are so close under the 1- and 2-order resonances: we have $\left(\gamma / \theta_{1}\right)^{2} \approx 10^{-4}$ and $\left(\gamma / \theta_{2}\right)^{2} \approx 0.25$, so the contributions of these terms are quite small.

\section{CONCLUSIONS}

In this work we obtained approximate analytical expressions for the dynamics of the nonstationary circuit QED system under the first- and second-order resonances. We considered a weak harmonic modulation of either the atomic transition frequency $(\Omega)$ or the atom-field coupling strength $(g)$ of a single or $N \gg 1$ identical qubits, demonstrating that all the phenomena that occur for the modulation frequency $\eta \sim 2 \omega_{0}$ (1-order resonances) also occur for $\eta \sim \omega_{0}$ (2-order resonances), where $\omega_{0}$ is the cavity frequency. It turned out that the modulation of $\Omega$ is more efficient for achieving the second-order resonances, in contrast to the first-order resonances for which both modulations produce similar transition rates. 
We solved numerically the 'standard master equation' of quantum optics to assess the feasibility of generating excitations from vacuum due to the counter-rotating terms under the second-order resonances. As demonstrated by figures 113, for small but realistic values of the dissipation parameters our proposal is realizable on the timescale of a few microseconds provided the modulation frequency is accurately tuned (with the absolute precision $\lesssim 10^{-5} \omega_{0}$ ). However, the benefit of lowering the modulation frequency by a factor of two is countered by the decrease of the transition rates by at least one order of magnitude, whence the dissipation plays a major role and partially destroys the oscillatory behavior expected from the unitary dynamics. Nevertheless, our results indicate that it is worth to pursue the second-order resonances in nonstationary circuit QED systems, as potential stronger modulation depths and atom-field coupling parameter could make them a viable mechanism of coherent selective coupling between the system dressed states.

\section{Acknowledgments}

ELSS acknowledges financial support by CAPES (Brazilian agency). AVD acknowledges a support of the Brazilian agency $\mathrm{CNPq}$ (Conselho Nacional de Desenvolvimento Científico e Tecnológico). We are grateful to L. C. Monteiro for some analytical evaluations at the initial stage of the work.

\section{Appendix A: Approximate expressions for $N \gg 1$}

For $N \gg 1$ identical noninteracting qubits we define the collective atomic operators via the Holstein-Primakoff transformation [56]

$$
\sum_{j=1}^{N} \hat{\sigma}_{+}^{(j)}=\hat{b}^{\dagger} \sqrt{N-\hat{b}^{\dagger} \hat{b}}, \sum_{j=1}^{N} \hat{\sigma}_{-}^{(j)}=\sqrt{N-\hat{b}^{\dagger} \hat{b}} \hat{b}, \sum_{j=1}^{N} \hat{\sigma}_{z}^{(j)}=2 \hat{b}^{\dagger} \hat{b}-N
$$

where the ladder operators $\hat{b}$ and $\hat{b}^{\dagger}$ satisfy the bosonic commutation relation. To the first order in $\hat{b}^{\dagger} \hat{b} / N$ the Hamiltonian (1) becomes $\hat{H}=\hat{H}_{J C}+\hat{H}_{G}+\hat{H}_{N G}+\hat{H}_{m}$, where

$$
\begin{aligned}
\hat{H}_{J C} & =\omega_{0} \hat{n}+\Omega_{0} \hat{b}^{\dagger} \hat{b}+\tilde{g}_{0}\left(\hat{a} \hat{b}^{\dagger}+\hat{a}^{\dagger} \hat{b}\right) \\
\hat{H}_{G} & =\tilde{g}\left(\hat{a} \hat{b}+\hat{a}^{\dagger} \hat{b}^{\dagger}\right)+i \chi_{0}\left(\hat{a}^{\dagger 2}-\hat{a}^{2}\right) \\
\hat{H}_{N G} & =-\frac{\tilde{g}}{2 N}\left(\hat{a}+\hat{a}^{\dagger}\right)\left(\hat{b}^{\dagger} \hat{b}+\hat{b}^{\dagger} \hat{b}^{2}\right) .
\end{aligned}
$$

$\hat{H}_{m}=\varepsilon_{\Omega} \sin (\eta t) \hat{b}^{\dagger} \hat{b}$ for the $\Omega$-modulation and $\hat{H}_{m}=\tilde{\varepsilon}_{g} \sin (\eta t)\left(\hat{a} \hat{b}^{\dagger}+\hat{a}^{\dagger} \hat{b}\right)$ for the $g$-modulation. We also defined the collective coupling parameter $\tilde{g} \equiv \sqrt{N} g \equiv \tilde{g}_{0}+\tilde{\varepsilon}_{g} f_{g}$.

We write the solution in the Heisenberg picture as [25, 26]

$$
\begin{aligned}
& \hat{a}=\beta^{-1} e^{-i t \Delta_{+} / 2}\left[\left(\beta_{+} \hat{A}_{h}+\tilde{g}_{0} \hat{B}_{h}\right) e^{-i t \beta / 2}+\left(\beta_{-} \hat{A}_{h}-\tilde{g}_{0} \hat{B}_{h}\right) e^{i t \beta / 2}\right] \\
& \hat{b}=\beta^{-1} e^{-i t \Delta_{+} / 2}\left[\left(\beta_{-} \hat{B}_{h}+\tilde{g}_{0} \hat{A}_{h}\right) e^{-i t \beta / 2}+\left(\beta_{+} \hat{B}_{h}-\tilde{g}_{0} \hat{A}_{h}\right) e^{i t \beta / 2}\right],
\end{aligned}
$$

where $\beta \equiv \sqrt{\Delta_{-}^{2}+4 \tilde{g}_{0}^{2}}, \beta_{ \pm}=\left(\beta \pm \Delta_{-}\right) / 2$ and the independent operators $\hat{A}_{h}, \hat{B}_{h}$ also satisfy the bosonic commutation relations. Next we propose the ansatz (for $k=\Omega, g$ )

$$
\begin{gathered}
\hat{A}_{h}=\exp \left[i \mathcal{F}_{A}^{(k)}\right] \times\left[\hat{A} e^{i t\left(\tilde{\delta}_{+}+\delta_{\chi}\right)}+i\left(\mathcal{F}_{A B}^{(k)}+\mathcal{F}_{2}^{(k)}\right) \hat{B} e^{i t \tilde{\delta}_{+}}\right] \\
\hat{B}_{h}=\exp \left[i \mathcal{F}_{B}^{(k)}\right] \times\left[\hat{B} e^{i t \tilde{\delta}_{+}}+i\left(\mathcal{F}_{A B}^{(k) *}+\mathcal{F}_{2}^{(k) *}\right) \hat{A} e^{i t\left(\tilde{\delta}_{+}+\delta_{\chi}\right)}\right],
\end{gathered}
$$

where small time-dependent c-number functions are:

$$
\mathcal{F}_{A}^{(\Omega)}=\varepsilon_{\Omega} \frac{\tilde{g}_{0}^{2}}{2 \beta^{2}}\left[2 \frac{e^{i t \eta}-1}{\eta}-\frac{e^{i t(\eta+\beta)}-1}{\eta+\beta}-\frac{e^{i t(\eta-\beta)}-1}{\eta-\beta}+\text { c.c. }\right]
$$




$$
\begin{aligned}
& \mathcal{F}_{A}^{(g)}=\tilde{\varepsilon}_{g} \frac{\tilde{g}_{0} \Delta_{-}}{2 \beta^{2}}\left[2 \frac{e^{i t \eta}-1}{\eta}-\frac{e^{i t(\eta+\beta)}-1}{\eta+\beta}-\frac{e^{i t(\eta-\beta)}-1}{\eta-\beta}+c . c .\right] \\
& \mathcal{F}_{B}^{(\Omega)}=\varepsilon_{\Omega} \frac{\tilde{g}_{0}^{2}}{2 \beta^{2}}\left[\left(2+\frac{\Delta_{-}^{2}}{\tilde{g}_{0}^{2}}\right) \frac{e^{i t \eta}-1}{\eta}+\frac{e^{i t(\eta+\beta)}-1}{\eta+\beta}+\frac{e^{i t(\eta-\beta)}-1}{\eta-\beta}+c . c .\right] \\
& \mathcal{F}_{B}^{(g)}=\tilde{\varepsilon}_{g} \frac{\tilde{g}_{0} \Delta_{-}}{2 \beta^{2}}\left[-2 \frac{e^{i t \eta}-1}{\eta}+\frac{e^{i t(\eta+\beta)}-1}{\eta+\beta}+\frac{e^{i t(\eta-\beta)}-1}{\eta-\beta}+\text { c.c. }\right] \\
& \mathcal{F}_{A B}^{(\Omega)}=\varepsilon_{\Omega} \frac{\tilde{g}_{0}}{2 \beta^{2}} \sum_{\mathcal{S}= \pm}\left[-\Delta_{-} \frac{e^{\mathcal{S} i t \eta}-1}{\eta}+\beta_{+} \frac{e^{\mathcal{S} i t(\eta+\mathcal{S} \beta)}-1}{\eta+\mathcal{S} \beta}-\beta_{-} \frac{e^{\mathcal{S i t}(\eta-\mathcal{S} \beta)}-1}{\eta-\mathcal{S} \beta}\right] \\
& \mathcal{F}_{A B}^{(g)}=\tilde{\varepsilon}_{g} \frac{\Delta_{-}}{2 \beta^{2}} \sum_{\mathcal{S}= \pm}\left[4 \delta_{-} \frac{e^{\mathcal{S} i t \eta}-1}{\eta}+\beta_{+} \frac{e^{\mathcal{S} i t(\eta+\mathcal{S} \beta)}-1}{\eta+\mathcal{S} \beta}-\beta_{-} \frac{e^{\mathcal{S} i t(\eta-\mathcal{S} \beta)}-1}{\eta-\mathcal{S} \beta}\right] \\
& \mathcal{F}_{2}^{(\Omega)}=\varepsilon_{\Omega} \frac{\tilde{g}_{0}}{2 \beta^{2}} \int_{0}^{t} d \tau\left[\mathcal{F}_{A}^{(\Omega)}(\tau)-\mathcal{F}_{B}^{(\Omega)}(\tau)\right] \\
& \times\left(\sum_{\mathcal{S}= \pm}\left[-\Delta_{-} e^{\mathcal{S} i \tau \eta}+\beta_{+} e^{\mathcal{S} i \tau(\eta+\mathcal{S} \beta)}-\beta_{-} e^{\mathcal{S} i \tau(\eta-\mathcal{S} \beta)}\right]-\text { c.c. }\right) \\
& \mathcal{F}_{2}^{(g)}=\tilde{\varepsilon}_{g} \frac{\Delta_{-}}{2 \beta^{2}} \int_{0}^{t} d \tau\left[\mathcal{F}_{A}^{(g)}(\tau)-\mathcal{F}_{B}^{(g)}(\tau)\right] \\
& \times\left(\sum_{\mathcal{S}= \pm}\left[4 \delta_{-} e^{\mathcal{S} i \tau \eta}+\beta_{+} e^{\mathcal{S} i \tau(\eta+\mathcal{S} \beta)}-\beta_{-} e^{\mathcal{S} i \tau(\eta-\mathcal{S} \beta)}\right]-c . c .\right) .
\end{aligned}
$$

One can check that to the first order in $\varepsilon_{\Omega}$ and $\tilde{\varepsilon}_{g}$ the slowly-varying operators $\hat{A}$ and $\hat{B}$ also satisfy the bosonic commutation relations.

Under resonant modulations the new operators $\hat{A}$ and $\hat{B}$ evolve according to the effective Hamiltonians $\hat{H}_{1}^{(k)}$ and $\hat{H}_{2}^{(k)}$ for the 1- and 2-order resonances, respectively. The approximate expressions for $\hat{H}_{1,2}^{(k)}$ are given below in the resonant and dispersive regimes. For the sake of clarity we omit the non-Gaussian (quartic) contributions arising from $\hat{H}_{N G}$ in equation A2 , as such terms were studied thoroughly in [26] for the 1-order resonances.

\section{Resonant regime}

In the regime $\Delta_{-}=0$ we have (for the sake of space, in all the expressions below we omit the hermitian conjugate on the right-hand side of the expressions):

- for $\eta \simeq 2 \omega_{0}$ or $\eta \simeq \omega_{0}$

$$
\begin{gathered}
\hat{H}_{1}^{(\Omega)} \simeq i \tilde{g}_{0} \frac{\tilde{g}_{0}}{2 \omega_{0}}\left(\frac{\varepsilon_{\Omega}}{4 \Omega_{0}}\right)\left[\hat{A}^{2} e^{2 i t \delta_{\chi}}-\hat{B}^{2}\right] e^{-i t\left(2 \omega_{0}-2 \tilde{\delta}_{+}-\eta\right)} \\
\hat{H}_{2}^{(\Omega)} \simeq-\tilde{g}_{0} \frac{\tilde{g}_{0}}{2 \omega_{0}}\left(\frac{\varepsilon_{\Omega}}{4 \Omega_{0}}\right)^{2} 8\left[\hat{A}^{2} e^{2 i t \delta_{\chi}}-\hat{B}^{2}\right] e^{-i t 2\left(\omega_{0}-\tilde{\delta}_{+}-\eta\right)} \\
\hat{H}_{1}^{(g)} \simeq 0, \hat{H}_{2}^{(g)} \simeq 0
\end{gathered}
$$


- for $\eta \simeq 2\left(\omega_{0} \pm \tilde{g}_{0}\right)$ or $\eta \simeq \omega_{0} \pm \tilde{g}_{0}$

$$
\begin{gathered}
\hat{H}_{1}^{(\Omega)} \simeq i \tilde{g}_{0}\left(\frac{\varepsilon_{\Omega}}{8 \Omega_{0}}\right)\left[\hat{A} \hat{B} e^{i t \delta_{\chi}} \pm \frac{1}{2} \hat{A}^{2} e^{2 i t \delta_{\chi}} \pm \frac{1}{2} \hat{B}^{2}\right] e^{-i t\left(2 \omega_{0} \pm 2 \tilde{g}_{0}-2 \tilde{\delta}_{+}-\eta\right)} \\
\hat{H}_{2}^{(\Omega)} \simeq-\tilde{g}_{0}\left(\frac{\varepsilon_{\Omega}}{8 \Omega_{0}}\right)^{2} \frac{1}{4}\left[14 \hat{A} \hat{B} e^{i t \delta_{\chi}} \pm 5 \hat{A}^{2} e^{2 i t \delta_{\chi}} \pm 5 \hat{B}^{2}\right] e^{-i t 2\left(\omega_{0} \pm \tilde{g}_{0}-\tilde{\delta}_{+}-\eta\right)} \\
\hat{H}_{1}^{(g)} \simeq-i \tilde{g}_{0} \frac{1}{2}\left(\frac{\tilde{\varepsilon}_{g}}{2 \tilde{g}_{0}}\right)\left[\hat{A} \hat{B} e^{i t \delta_{\chi}} \pm \frac{1}{2} \hat{A}^{2} e^{2 i t \delta_{\chi}} \pm \frac{1}{2} \hat{B}^{2}\right] e^{-i t\left(2 \omega_{0} \pm 2 \tilde{g}_{0}-2 \tilde{\delta}_{+}-\eta\right)} \\
\hat{H}_{2}^{(g)} \simeq \tilde{g}_{0} \frac{1}{2}\left(\frac{\tilde{\varepsilon}_{g}}{2 \tilde{g}_{0}}\right)^{2} \frac{2 \tilde{g}_{0}}{\omega_{0}}\left[ \pm \hat{A} \hat{B} e^{i t \delta_{\chi}}+\frac{1}{2} \hat{A}^{2} e^{2 i t \delta_{\chi}}+\frac{1}{2} \hat{B}^{2}\right] e^{-i t 2\left(\omega_{0} \pm \tilde{g}_{0}-\tilde{\delta}_{+}-\eta\right)} .
\end{gathered}
$$

\section{Dispersive regime}

In the regime $\left|\Delta_{-}\right| / 2 \gg\left|\tilde{g}_{0}\right|$ we have

- for $\eta \simeq \Delta_{+}$or $\eta \simeq \Delta_{+} / 2$ (AJC-like behavior)

$$
\begin{gathered}
\hat{H}_{1}^{(\Omega)} \simeq i \tilde{g}_{0}\left(\frac{\varepsilon_{\Omega}}{2 \Delta_{+}}\right)\left[\hat{A} \hat{B} e^{i t \delta_{\chi}}-\frac{\tilde{g}_{0}}{\Delta_{-}} \hat{A}^{2} e^{2 i t \delta_{\chi}}+\frac{\tilde{g}_{0}}{\Delta_{-}} \hat{B}^{2}\right] e^{-i t\left(\Delta_{+}-2 \tilde{\delta}_{+}-\eta\right)} \\
\hat{H}_{2}^{(\Omega)} \simeq-\tilde{g}_{0}\left(\frac{\varepsilon_{\Omega}}{2 \Delta_{+}}\right)^{2} 2\left[\hat{A} \hat{B} e^{i t \delta_{\chi}}-\frac{\tilde{g}_{0}}{\Delta_{-}} \hat{A}^{2} e^{2 i t \delta_{\chi}}+\frac{\tilde{g}_{0}}{\Delta_{-}} \hat{B}^{2}\right] e^{-i t\left(\Delta_{+}-2 \tilde{\delta}_{+}-2 \eta\right)} \\
\hat{H}_{1}^{(g)} \simeq-i \tilde{g}_{0}\left(\frac{\tilde{\varepsilon}_{g}}{2 \tilde{g}_{0}}\right)\left[\hat{A} \hat{B} e^{i t \delta_{\chi}}-\frac{\tilde{g}_{0}}{\Delta_{-}} \hat{A}^{2} e^{2 i t \delta_{\chi}}+\frac{\tilde{g}_{0}}{\Delta_{-}} \hat{B}^{2}\right] e^{-i t\left(\Delta_{+}-2 \tilde{\delta}_{+}-\eta\right)} \\
\hat{H}_{2}^{(g)} \simeq-\tilde{g}_{0}\left(\frac{\tilde{\varepsilon}_{g}}{2 \tilde{g}_{0}}\right)^{2}\left(\frac{2 \tilde{g}_{0}}{\Delta_{+}}\right)^{2} 5\left[\hat{A} \hat{B} e^{i t \delta_{\chi}}-\frac{\tilde{g}_{0}}{\Delta_{-}} \hat{A}^{2} e^{2 i t \delta_{\chi}}+\frac{\tilde{g}_{0}}{\Delta_{-}} \hat{B}^{2}\right] e^{-i t\left(\Delta_{+}-2 \tilde{\delta}_{+}-2 \eta\right)} ;
\end{gathered}
$$

- for $\eta \simeq 2 \omega_{0}$ or $\eta \simeq \omega_{0}$ (DCE behavior $)$

$$
\begin{gathered}
\hat{H}_{1}^{(\Omega)} \simeq i \tilde{\delta}_{-} \frac{\Omega_{0}}{\Delta_{+}}\left(\frac{\varepsilon_{\Omega}}{2 \Omega_{0}}\right)\left[\hat{A}^{2} e^{2 i t \delta_{\chi}}+\frac{2 \tilde{g}_{0}}{\Delta_{-}} \hat{A} \hat{B} e^{i t \delta_{\chi}}+\frac{\tilde{g}_{0}^{2}}{\Delta_{-}^{2}} \hat{B}^{2}\right] e^{-i t\left(2 \omega_{0}+2 \tilde{\delta}_{-}-2 \tilde{\delta}_{+}-\eta\right)} \\
\hat{H}_{2}^{(\Omega)} \simeq-\tilde{\delta}_{-} \frac{\Omega_{0}}{\Delta_{+}}\left(\frac{\varepsilon_{\Omega}}{2 \Omega_{0}}\right)^{2}\left[\hat{A}^{2} e^{2 i t \delta_{\chi}}+\frac{4 \tilde{g}_{0}}{\Delta_{-}} \hat{A} \hat{B} e^{i t \delta_{\chi}}+\frac{3 \tilde{g}_{0}^{2}}{\Delta_{-}^{2}} \hat{B}^{2}\right] e^{-i t 2\left(\omega_{0}+\tilde{\delta}_{-}-\tilde{\delta}_{+}-\eta\right)} \\
\hat{H}_{1}^{(g)} \simeq-i \frac{2 \tilde{\delta}_{-} \Omega_{0}}{\Delta_{+}}\left(\frac{\tilde{\varepsilon}_{g}}{2 \tilde{g}_{0}}\right)\left[\hat{A}^{2} e^{2 i t \delta_{\chi}}+\frac{2 \tilde{g}_{0}}{\Delta_{-}} \hat{A} \hat{B} e^{i t \delta_{\chi}}+\frac{\tilde{g}_{0}^{2}}{\Delta_{-}^{2}} \hat{B}^{2}\right] e^{-i t\left(2 \omega_{0}+2 \tilde{\delta}_{-}-2 \tilde{\delta}_{+}-\eta\right)} \\
\hat{H}_{2}^{(g)} \simeq \frac{2 \tilde{\delta}_{-} \Omega_{0}}{\Delta_{+}}\left(\frac{\tilde{\varepsilon}_{g}}{2 \tilde{g}_{0}}\right)^{2} \frac{\Delta_{+} \Delta_{-}}{2 \Omega_{0}^{2}}\left[\hat{A}^{2} e^{2 i t \delta_{\chi}}+\frac{2 \tilde{g}_{0}}{\Delta_{-}} \hat{A} \hat{B} e^{i t \delta_{\chi}}+\frac{\tilde{g}_{0}^{2}}{\Delta_{-}^{2}} \hat{B}^{2}\right] e^{-i t 2\left(\omega_{0}+\tilde{\delta}_{-}-\tilde{\delta}_{+}-\eta\right)} ;
\end{gathered}
$$


- for $\eta \simeq 2 \Omega_{0}$ or $\eta \simeq \Omega_{0}$ we have an effect analogous to DCE, but for the collective atomic excitations (so it was called Inverse-DCE in [26])

$$
\begin{gathered}
\hat{H}_{1}^{(\Omega)} \simeq-i \tilde{\delta}_{-} \frac{\omega_{0}}{\Delta_{+}}\left(\frac{\varepsilon_{\Omega}}{2 \Omega_{0}}\right)\left[\hat{B}^{2}-\frac{2 \tilde{g}_{0}}{\Delta_{-}} \hat{A} \hat{B} e^{i t \delta_{\chi}}+\frac{\tilde{g}_{0}^{2}}{\Delta_{-}^{2}} \hat{A}^{2} e^{2 i t \delta_{\chi}}\right] e^{-i t\left(2 \Omega_{0}-2 \tilde{\delta}_{-}-2 \tilde{\delta}_{+}-\eta\right)} \\
H_{2}^{(\Omega)} \simeq \tilde{\delta}_{-} \frac{\omega_{0}}{\Delta_{+}}\left(\frac{\varepsilon_{\Omega}}{2 \Omega_{0}}\right)^{2}\left[\hat{B}^{2}-\frac{4 \tilde{g}_{0}}{\Delta_{-}} \hat{A} \hat{B} e^{i t \delta_{\chi}}+\frac{3 \tilde{g}_{0}^{2}}{\Delta_{-}^{2}} \hat{A}^{2} e^{2 i t \delta_{\chi}}\right] e^{-i t 2\left(\Omega_{0}-\tilde{\delta}_{-}-\tilde{\delta}_{+}-\eta\right)} \\
\hat{H}_{1}^{(g)} \simeq i \frac{2 \tilde{\delta}_{-} \omega_{0}}{\Delta_{+}}\left(\frac{\tilde{\varepsilon}_{g}}{2 \tilde{g}_{0}}\right)\left[\hat{B}^{2}-\frac{2 \tilde{g}_{0}}{\Delta_{-}} \hat{A} \hat{B} e^{i t \delta_{\chi}}+\frac{\tilde{g}_{0}^{2}}{\Delta_{-}^{2}} \hat{A}^{2} e^{2 i t \delta_{\chi}}\right] e^{-i t\left(2 \Omega_{0}-2 \tilde{\delta}_{-}-2 \tilde{\delta}_{+}-\eta\right)} \\
\hat{H}_{2}^{(g)} \simeq \frac{2 \tilde{\delta}_{-} \omega_{0}}{\Delta_{+}}\left(\frac{\tilde{\varepsilon}_{g}}{2 \tilde{g}_{0}}\right)^{2} \frac{\Delta_{+} \Delta_{-}}{2 \omega_{0}^{2}}\left[\hat{B}^{2}-\frac{2 \tilde{g}_{0}}{\Delta_{-}} \hat{A} \hat{B} e^{i t \delta_{\chi}}+\frac{\tilde{g}_{0}^{2}}{\Delta_{-}^{2}} \hat{A}^{2} e^{2 i t \delta_{\chi}}\right] e^{-i t 2\left(\Omega_{0}-\tilde{\delta}_{-}-\tilde{\delta}_{+}-\eta\right)} .
\end{gathered}
$$

One can see that under the second-order resonances the $g$-modulation always yields transition rates at least one order of magnitude smaller than the $\Omega$-modulation. Lastly, Anti-DCE for light and matter excitations (or AntiInverse-DCE) can be derived from the Non-Gaussian Hamiltonian $\hat{H}_{N G}$ [26, 45, however we do not present the results because under the 2-order resonances the corresponding transition rates are very small (see section IIB for the case $N=1)$.

[1] Dodonov V V 2001 Adv. Chem. Phys. 119309

[2] Dodonov V V 2010 Phys. Scr. 82038105

[3] Dalvit D A R, Maia Neto P A and Mazzitelli F D 2011 Casimir Physics (Lecture Notes in Physics vol 834) ed D Dalvit, P Milonni, D Roberts and F da Rosa (Berlin: Springer) p 419

[4] Nation P D, Johansson J R, Blencowe M P and Nori F 2012 Rev. Mod. Phys. 841

[5] Fulling S A and Davies P C W 1976 Proc. R. Soc. A 348393

[6] Barton G and Eberlein C 1993 Ann. Phys. (NY) 227222

[7] Maia Neto P A and Machado L A S 1996 Phys. Rev. A 543420

[8] Moore G T 1970 J. Math. Phys. 112679

[9] Law C K 1994 Phys. Rev. A 49433

[10] Dodonov V V 1995 Phys. Lett. A 207126

[11] Lambrecht A, Jaekel M-T and Reynaud S 1996 Phys. Rev. Lett. 77615

[12] Carusotto I, Balbinot R, Fabbri A and Recati A 2010 Eur. Phys. J. D 56391

[13] Jaskula J C, Partridge G B, Bonneau M, Lopes R, Ruaudel J, Boiron D and Westbrook C I 2012 Phys. Rev. Lett. 109 220401

[14] Busch X, Carusotto I and Parentani R 2014 Phys. Rev. A 89043819

[15] Dodonov V V and Mendonça J T 2014 Phys. Scr. T160 014008

[16] Blais A, Huang R-S, Wallraff A, Girvin S M and Schoelkopf R J 2004 Phys. Rev. A 69062320

[17] Wallraff A, Schuster D I, Blais A, Frunzio L, Huang R-S, Majer J, Kumar S, Girvin S M and Schoelkopf R J 2004 Nature 431162

[18] Chiorescu I, Bertet P, Semba K, Nakamura Y, Harmans C J P M and Mooij J E 2004 Nature 431159

[19] Schuster D I et al. 2007 Nature 445515

[20] Schoelkopf R J and Girvin S M 2008 Nature 451664

[21] Wilson C M, Johansson G, Pourkabirian A, Simoen M, Johansson J R, Duty T, Nori F and Delsing P 2011 Nature 479 376

[22] Lähteenmäki P, Paraoanu G S, Hassel J and Hakonen P J 2013 Proc. Nat. Acad. Sci. 1104234

[23] Dodonov A V 2009 J. Phys.: Conf. Ser. 161012029

[24] De Liberato S, Gerace D, Carusotto I and Ciuti C 2009 Phys. Rev. A 80053810

[25] Dodonov A V 2013 J. Phys. A 47285303

[26] de Sousa I M and Dodonov A V 2015 J. Phys. A 48245302

[27] Veloso D S and Dodonov A V 2015 J. Phys. B 48165503

[28] Dodonov A V, Militello B, Napoli A and Messina A 2016 Phys. Rev. A 93052505

[29] Makhlin Yu, Schon G and Shnirman A 2001 Rev. Mod. Phys. 73357

[30] You J Q and Nori F 2011 Nature 474589 
[31] Devoret M H and Schoelkopf R J 2013 Science 3391169

[32] Clarke J and Wilhelm F K 2008 Nature 4531031

[33] Majer J et al. 2007 Nature 449443

[34] Srinivasan S J, Hoffman A J, Gambetta J M and Houck A A 2011 Phys. Rev. Lett. 106083601

[35] Chen Yu et al. 2014 Phys. Rev. Lett. 113220502

[36] Zeytinoğlu S, Pechal M, Berger S, Abdumalikov Jr. A A, Wallraff A and Filipp S 2015 Phys. Rev. A 91043846

[37] Kirchmair G et al. 2013 Nature 495205

[38] Felicetti S, Sabín C, Fuentes I, Lamata L, Romero G and Solano E 2015 Phys. Rev. B 92064501

[39] Benenti G, D'Arrigo A, Siccardi S and Strini G 2014 Phys. Rev. A 90052313

[40] Felicetti S, Sanz M, Lamata L, Romero G, Johansson G, Delsing P and Solano E 2014 Phys. Rev. Lett. 113 093602

[41] Stassi R, De Liberato S, Garziano L, Spagnolo B and Savasta S 2015 Phys. Rev. A 92013830

[42] Rossatto D Z, Felicetti S, Eneriz H, Rico E, Sanz M and Solano E 2016 Phys. Rev. B 93094514

[43] Dodonov A V and Dodonov V V 2012 Phys. Rev. A 86015801

[44] Dodonov A V 2013 Phys. Scr. 87038103

[45] Monteiro L C and Dodonov A V 2016 Phys. Lett. A 3801542

[46] Ristè D et al. 2013 Nature 502350

[47] Sun L et al. 2014 Nature $\mathbf{5 1 1} 444$

[48] Rabi I I 1936 Phys. Rev. 49324

[49] Rabi I I 1937 Phys. Rev. 51652

[50] Braak D 2011 Phys. Rev. Lett. 107100401

[51] Schleich W P 2001 Quantum Optics in Phase Space (Berlin: Wiley)

[52] Malekakhlagh M and Türeci H E 2016 Phys. Rev. A 93012120

[53] Beaudoin F, Gambetta J M and Blais A 2011 Phys. Rev. A 84043832

[54] André A, DeMille D, Doyle J M, Lukin M D, Maxwell S E, Rabl P, Schoelkopf R J and Zoller P 2006 Nat. Phys. 2 636

[55] Carr L D, DeMille D, Krems R V and Ye J 2009 New J. Phys. 11, 055049

[56] Garraway B M 2011 Phil. Trans. R. Soc. A 3691137

[57] Vogel W and Welsch D -G 2006 Quantum Optics (Berlin: Wiley) 